\title{
ANTROPOLOGÍA DEL EMBARAZO, PARTO Y PUERPERIO EN LA CIUDAD DE ELCHE
}

\author{
Salazar Agulló, M.; Navarro Pellicer, S.; González Rodríguez, M. \\ Matronas Residentes. Hospital Gral. Universitario de Elche.
}

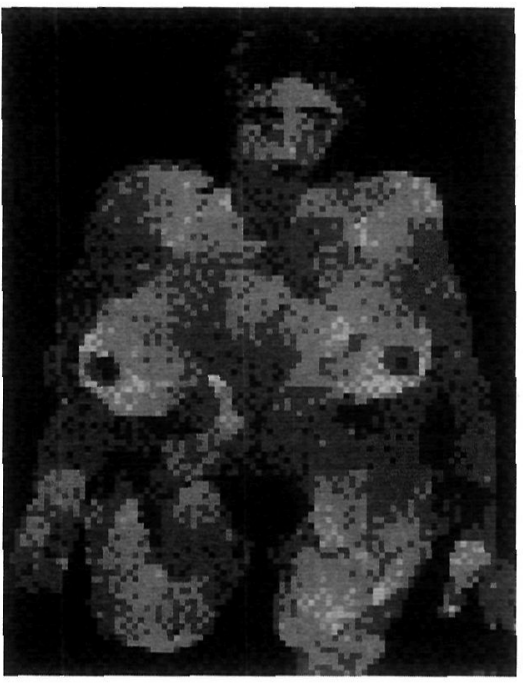

Sarah Gee «Desnudo azul»

\section{RESUMEN}

El objetivo del estudio ha sido conocer las actitudes que sobre el embarazo, parto y puerperio tenían mujeres residentes en la comarca de Elche, que hubieran tenido hijos hacía más de veinte años. Se entrevistaron a treinta, con edades comprendidas entre los 48 y 87 años (media de 66,4 años); la media de partos fue de 4,6 y el nivel sociocultural era bajo; más de la mitad procedían de lugares de fuera de la comarca pero llevaban viviendo en la zona una media de 46 años. La metodología utilizada fue la entrevista semi-estructurada, utilizando un cuestionario ya validado; las entrevistas se grabaron y posteriormente transcribieron, extrayendo de todas ellas las conclusiones, de las que cabe destacar que las mujeres de nuestro estudio tenían en común: gran número de embarazos; otorgaban gran importancia al tema de la maternidad; tuvieron partos domiciliarios y en la actualidad les inspira más confianza el parto hospitalario; son partidarias de que exista un distanciamiento entre los embarazos pese a que ellas no los tuvieron; no recibieron controles sanitarios; describen a la matrona como la profesional que las atendía en el parto y en el puerperio y quien les bañaba al niño; no creen en la influencia de los astros ni en símbolos religiosos.

\section{ANTHROPOLOGY OF PREGNANCY, LABOUR AND POST-LABOUR IN THE CITY OF ELCHE}

\begin{abstract}
The present study was prepared in Elche, in the first part of 1997. The aim was to discover what had been the attitudes towards pregnancy, labour and post-labour of women who had been mothers twenty years earlier. 30 samples were analysed, covering ages between 48 and 87 (average 66.4 years old). The average of child-births was 4.6 and the socio-cultural level was rather low. More than half of the sample mothers were from the outskirts of Elche but had been living in the area for more than 46 years.

The method used was a semi-structured interview, with a questionaire already validated. Interviews were recorded and then transcribed. The conclusions were: the sample women had in common a great number of pregnancies. For them all maternity was very important. Most of them were simply house-wives whose main task was «house-work» and perhaps incidentally they helped their husbands. Most of them have given birth at home although they nowadays feel it is safer to give birth in hospitals. Their present feeling is that mothers should not have the number of children they had had, and time between preganancies should have been longer. They had not received sanitary or health control in their pregnancies. Their reference was always the midwife as the professional in charge of their labour and post-labour, as well as to care for the baby during that period. They neither believe in astrological influence towards the vital process nor do they show faith in religious symbols in the same way.
\end{abstract}




\section{INTRODUCCIÓN}

Cuando estamos próximos a iniciar un nuevo milenio y echamos la vista atrás, observamos los grandes cambios que se han producido en nuestro medio: el parto ya no es una de las principales causas de muerte de la mujer; se acepta que la reproducción es un derecho antes que una obligación y que los deberes que conlleva (anticoncepción o crianza), se entienden mejor desde la autoestima femenina, el poder personal y la responsabilidad social. Antes de desarrollar nuestro trabajo, haremos una breve reseña de cómo ha sido la atención al parto a lo largo de la historia:

En las sociedades primitivas las mujeres embarazadas daban a luz solas o, en todo caso, ayudadas por las ancianas de la tribu. Es en la medicina griega cuando aparecen las comadronas, organizadas por Hipócrates para atender a las mujeres en los partos. Esta situación persiste hasta que en los siglos XVII y XVIII empiezan a intervenir los médicos, pero su atención se prestaba inicialmente a mujeres de clases acomodadas. El parto, hasta entonces un acto natural, comienza a ser un acto médico.

A mediados del siglo pasado el $10 \%$ de embarazadas morían en el parto y otro $10 \%$ tenía complicaciones puerperales. Pero con el desarrollo de la Medicina y de los instrumentos técnicos, se produce una disminución de la mortalidad tanto materna como fetal. El embarazo lo entendemos como un proceso donde se pone en juego no sólo lo biológico, sino también lo psicológico (a través de la singularidad de la mujer) y lo social, por lo que representan el embarazo, el parto y la maternidad en nuestra sociedad y por el papel que tiene la mujer en la misma.

Cuando nos propusimos realizar este estudio se produjo una reacción unánime: trataríamos de realizar la encuesta a mujeres a las que por su edad no les resultase extraño el parto a domicilio, con la convicción de que poseerían aún en su haber muchos conocimientos, creencias y técnicas que podían haber caído en desuso y que nosotras podríamos rescatar de su memoria (y poder pasar a la información escrita), no desapareciendo cuando ellas lo hiciesen. Estos conocimientos han servido para incrementar nuestra formación sobre este aspecto vital, marco en el cual vamos a desarrollar nuestra actividad laboral.

Ahora que los avances científicos permiten parir sin sufrir dolor, tener hijos después de los límites biológicos (no son infrecuentes casos de madres con 50 años y más años) e incluso aspirar a tener bebés con cierto diseño genético, nos hemos permitido asomarnos a un pasado muy reciente, lleno de curiosidades y detalles de los que se podrán extraer una serie de conclusiones, que, para comenzar, a nosotras ya nos están sirviendo para entender mejor el presente.

\section{Características de la población de estudio}

La ciudad de Elche, situada al sur de la Comunidad Valenciana, es en cuanto a número de habitantes (191.812, según el Padrón de 1-5-1996) la tercera ciudad de la misma. Su término municipal es de $326 \mathrm{Km}^{2}$ de superficie, con una densidad de población de $588 \mathrm{hab} / \mathrm{Km}^{2}$. La tasa de natalidad sigue siendo una de las más altas de la Comunidad Valenciana, y sólo está superada por la de la Vega Baja. La actividad económica por excelencia es la industria del calzado que tuvo su máximo esplendor en los años 60; fue un verdadero foco de atracción para miles de inmigrantes del centro y sur de España (CastillaLa Mancha, Extremadura y Andalucía), lo que motivó un importante crecimiento demográfico que ha repercutido de forma importante en muchos aspectos culturales. Sanitariamente está adscrita al Area de Salud no 19 y posee límites equiparables a la comarca natural del campo de Elche (Baix Vinalopó) con una superficie total de 487 $\mathrm{Km}^{2}$ y una población cercana a los 230.000 habitantes.

En la actualidad coexisten zonas donde la atención sanitaria se presta en centros de Salud (Crevillente, Santa Pola y en la ciudad de Elche los centros de Altabix, Jo-

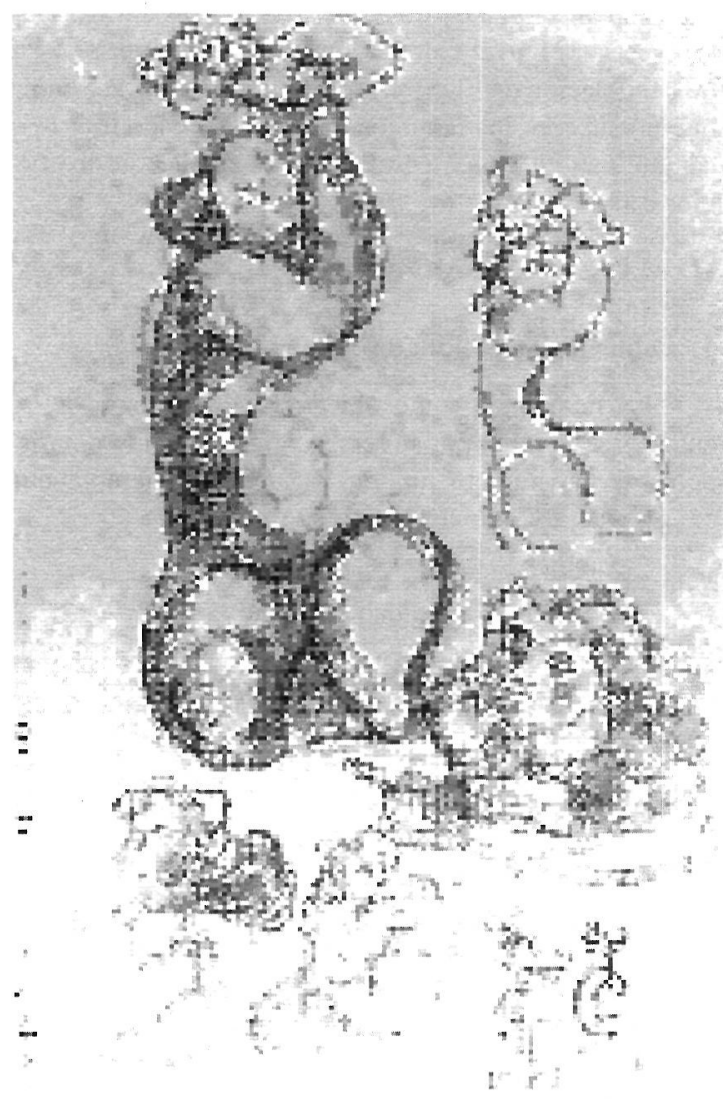

I Love My Baby 1941 - crayon on paper • Chaim Gross 
sé $\mathrm{M}^{\mathrm{a}}$ Pemán, Carrús y recientemente $\mathrm{El}$ Toscar), pero aún queda dentro del límite urbano una importante zona donde persiste el modelo tradicional (próximo al 50\%) y que recibe las prestaciones sanitarias en el Ambulatorio de San Fermín.

Pese a disponer de una de las más altas tasas de natalidad, como se ha comentado, las pirámides de población de los últimos años revelan una tendencia clara al envejecimiento, ya que la población mayor de 65 años se sitúa en torno al $15 \%$. Como en la mayoría de las poblaciones españolas, en nuestra ciudad el lugar de la atención al parto ha sido tradicionalmente el propio domicilio, tendencia que en Elche se mantuvo hasta casi entrados los años 70. La cobertura hospitalaria hasta entonces se concentraba en la ciudad de Alicante que dista sólo 20 $\mathrm{Km}$., aunque quedaba reservada la atención obstétrica para mujeres con patologías (cardiópatas, diabéticas, etc.). En esas fechas Elche disponía de dos clínicas privadas (con baja dotación de camas) que fundamentalmente eran obstétricas: «Virgen de la Asunción» (Dr. Morenilla) y «Sagrada Familia» (Dr. Bonete), y a las que accedían las mujeres con suficiencia económica. La apertura del Hospital de Elche en el año 1978 modifica casi radicalmente los hábitos de la atención obstétrica. Hay que reseñar el nivel cultural; en la actualidad un $87,72 \%$ de la población de Elche presenta unos niveles de estudio que no superan los Estudios Primarios; siendo este porcentaje más relevante en el rango de población que hemos estudiado.

\section{MATERIAL Y MÉTODO}

\section{Población seleccionada}

Nos marcamos como objetivo la realización de 30 encuestas, no tanto por su representatividad sino por disponer de un número mínimo y adecuado siempre que cumplieran los siguientes requisitos:

- ser mujer

- residir en la actualidad en Elche

- tener como mínimo un hijo

- haber dado a luz cuando la práctica habitual era el parto domiciliario.

Se determinaron estos criterios por la accesibilidad y por la supuesta riqueza de experiencias vividas no medicalizadas, y que por los avances tecnológicos están hoy en desuso, aspectos que pretendíamos conocer. Para conseguir este número se ha recurrido a familiares, personas cercanas e instituciones (públicas y privadas) a las que acuden mujeres que cumplen estos requisitos. A todas ellas se les explicaron los objetivos del estudio, solicitando su colaboración. No hubo ninguna negativa a participar en el mismo.

\section{Técnica}

La técnica utilizada ha sido la entrevista-participación, mediante el uso de un cuestionario semiestructurado con preguntas abiertas y cerradas; dicho cuestionario fue extraído de una parte del utilizado para el trabajo de «Fenómenos biológicos y fenómenos culturales: la interpretación popular del ciclo vital en dos comunidades alicantinas».

A través de la conversación abierta hemos obtenido información más precisa sobre comportamientos y actitudes sobre el objeto del trabajo. Se ha utilizado una grabadora con el propósito de registrar literalmente las respuestas.

\section{Cuestionario (Anexo I)}

El cuestionario utilizado consta de 47 preguntas de las cuales se distinguen cuatro grupos:

a) Datos generales (rasgos sociodemográficos) (preguntas 1 a 10)

b) Comportamientos, actitudes y creencias frente al embarazo (preguntas 11 a 32)

c) Comportamientos, actitudes y creencias frente al parto (preguntas 33 a 43)

d) Comportamientos, actitudes y creencias frente al puerperio (preguntas 44 a 47)

Tres encuestadoras recibieron la misma información previa sobre la metodología y pasaron las encuestas. Los resultados se analizaron en conjunto.

\section{Método}

Con los resultados obtenidos se ha realizado un estudio estadístico de carácter descriptivo compuesto por la distribución de frecuencias absolutas y porcentajes, así como por la representación gráfica de las preguntas cerradas más relevantes.

\section{RESULTADOS}

\section{III.A Rasgos socio-demográficos}

1.- EDAD: Las mujeres de nuestro estudio tienen una media de 66,4 años (DS), con un rango de edades comprendido entre 48 y 87 años.

\section{2.- FORMULA OBSTETRICA:}

Gestaciones: El rango está comprendido entre 1 y 16 , siendo la media de 5,2 embarazos, que se distribuyeron de la siguiente forma:

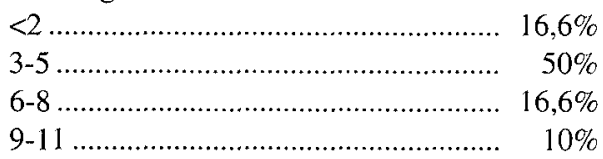




$$
+12 \text {. }
$$

$6,6 \%$

Abortos: Un tercio de las mujeres sufrieron al menos, un aborto, sumando en total 19 , siendo de destacar que sólo una de ellas tuvo 6.

Partos: la media fue de 4,6 por mujer. de 4,1

Hijos vivos en la actualidad: representan una media

\section{3.- LUGAR DE NACIMIENTO:}

1.- Pueblo donde se hace la encuesta ... 23\% (7)

2.- Otra población de la misma comarca $30 \% \quad$ (9)

3.- Otra población del País Valenciano. $13 \% \quad$ (4)

4.- Cualquier otra población .................. 33\% (10)

4.- SI NO ES DE LA LOCALIDAD, ¿CUÁNTOS AÑOS LLEVA VIVIENDO AQUÍ?

El rango oscila entre 8 y 79 años, con una media de 45,9 años.

\begin{tabular}{|c|c|}
\hline 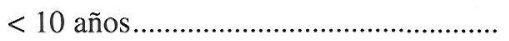 & $6,6 \%$ \\
\hline 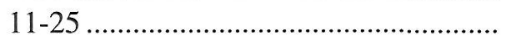 & $13 \%$ \\
\hline $26-50$ & $33 \%(10)$ \\
\hline
\end{tabular}

\section{5.- PROFESIÓN:}

0 - Sus labores. $60 \%(18)$

1.- Sector primario $23,3 \%$

$10 \%$

2.- Sector secundario

3.- Sector terciario

$6,6 \%$

\section{6.- ACTIVIDAD LABORAL:}

1.- Activa $33,3 \%(10)$

2.- Parada

3.- Retirada $63,3 \%$

\section{7.- NIVEL DE ESTUDIOS:}

1.- Analfabetos

2.- No estudios y no clasificable. $63,3 \%$

3.- Primarios

$20 \%$

4.- Secundarios

$6,6 \%$

5.- Superiores

\section{8.- PROFESIÓN DEL CABEZA DE FAMILIA:}

Sector primario (agricultura,

ganadería,...):

Sector secundario (Industria...) $36,6 \%$ (11)

Sector terciario (Servicios) $23,3 \% \quad(7)$

\section{9.- EL CABEZA DE FAMILIA ESTÁ:}

1.- Activo. $16,6 \%$

2.- Parado $6,6 \%$

3.- Retirado $53,3 \%(16)$

4.- Fallecido $23,3 \%$

10.- ADEMAS DEL CABEZA DE FAMILIA, EXISTE ALGUN OTRO MIEMBRO DE LA FAMILIA TRABAJANDO:

1.- Sí.

2.- No $50 \%$
III.B. Actitudes y creencias sobre el embarazo

11.- ¿CONSIDERA QUE SÓlO SE ESTÁ REALIZADA COMO MUJER CUANDO SE HA SIDO MADRE?

Sí $56,6 \%(17)$

No. $36,6 \%(11)$

$\mathrm{NS} / \mathrm{NC}$ $6,6 \%$

\section{¿POR QUE?}

SÍ:

- porque es natural que la mujer tenga hijos

- mejor que sea madre para ser mujer

- es lo más bonito del mundo

- es un privilegio

- si puedes, ¿por qué te vas a privar?

- «la mujer debe traer hijos al mundo».

- no me imagino sin hijos... 5

- los hijos dan la felicidad

- si no te quedas muy sola

- el matrimonio sin hijos no es lo mismo

NO:

- te haces más madura si tienes hijos.

- me he sentido más mujer cuando he sido madre.

- eso es una tontería.

- es importante pero no fundamental

- aunque es una experiencia que marca definitivamente a la mujer.

12.- SI UN MATRIMONIO NO PUEDE TENER HIJOS, ¿DE QUIEN ES LA CULPA?

- Del hombre 0

- De la mujer $10 \% \quad(3)$

- De los dos $80 \%(24)$

- NS/NC $6,6 \% \quad(2)$

- OTROS: Del demonio $3,3 \% \quad(1)$

¿POR QUE?

- De los dos:

- porque antes han hecho cosas para no tener

- es más culpa de la mujer porque aborta

- el fallo puede estar en uno o en otro

- antes decían que de la mujer, pero es de los dos.

- siempre se piensa primero en la mujer.

13.- ¿SABE DE ALGÚN REMEDIO PARA PODER TENER HIJOS CUANDO ÉSTOS TARDAN EN LLEGAR?

NO conoce ninguno $100 \%$

$\mathrm{y}$ algunos añaden:

- paciencia...

- ponerse en tratamiento

- la «piedra del olivino» de Lanzarote.

14.- ¿CONOCE ALGUN SANTO, VIRGEN O SANTUARIO A LA QUE SE ACUDA PARA TENER HIJOS? 
- No conoce $73,3 \%(22)$

- Cristo de la Columna $3,3 \%$

- San Francisco. $3,3 \%$

- San Ramón Nonato

$10 \%$

- Virgen de Cortes

$3,3 \%$

- Cristo de Zalamea

$3,3 \%$

15.- ¿CREE QUE LAS ESTRELLAS, EL SOL O LA LUNA TIENEN ALGUNA INFLUENCIA EN TENER O NO TENER HIJOS?

- No cree en ello.

- Según esté luna llena, creciente o menguante salía un chico o una chica.

- Cree que tiene influencia pero no sabe por qué.

16.- ¿CÓMO SE VAN FORMANDO LOS NIÑOS? ¿A PARTIR DE QUÉ?

- No saben explicarlo $36,6 \%$ (11)

- «De lo que los hombres despiden»

- «Del semen del hombre y óvulos de la mujer

- «Acostándose con la pareja» $5 \%$

- «A partir de los 40 d. por la sustancia del hombre (semilla) y la mujer».

- «Si es chico a los 40 días se forma, y si es chica a los tres meses».

- «De tener las reglas bien y estar limpia»

\section{7.- ¿QUÉ CREE QUE SON LOS ANTOJOS?}

- Cosas que te apetecen, deseos, algo que te encaprichas con mucha fuerza $60 \%$ (18)

- Tonterías. $26,6 \%$

- Llamar la atención del marido $6,6 \% \quad(2)$

- No sé lo que son $6,6 \%$

18.- ¿QUE OCURRE SI NO SE SATISFACEN LOS ANTOJOS?

- El niño nace con manchas con la forma del deseo $63,3 \%$

- Son tonterías $16,6 \%$

- Puedes abortar, malformaciones.

$20 \%$

19.- ¿CUÁLES SON LOS ANTOJOS QUE MAS FRECUENTEMENTE HA OÍDO?

- Productos alimenticios como frutas (moras, cerezas, uvas, melón, sandía), pasteles, chocolate, jamón ................ 76,6\%

- Flores $40 \%(12)$

- NS/NC. $10 \% \quad(3)$

- De todo, cualquier cosa $6,6 \% \quad(2)$

- «Excepto trabajo, he oído de todo»... 3,3\% (1)

20.- ¿CONOCE ALGÚN METODO POPULAR PARA SABER EL SEXO DEL FETO?

- No conoce ningún método. $30 \% \quad(9)$

- Sí conoce algún método. $70 \%(21)$

- Raspa de sardina en la lumbre y antes de que se queme se sale a la calle y el primer ser vivo que pase si es macho es un varón y si es hembra lo que llevas es una mujer.

- Si es chica se te hinchan boca, labios y se te mancha la cara y si es chico te pones fea.

- Se coge un pollo del pico, si voloteaba era chico y si se quedaba quieto era chica.

- Se coge una medalla y se le dan vueltas sobre la mano derecha abierta de la embarazada y según gire perpendicular o circular sobre la mano es chico o chica (5).

- Se ponían unas tijeras en una silla y en la otra un mechero; si la embarazada elegía la silla del mechero era un niño y si era la de las tijeras era una niña.

- Según el ritmo de las pulsaciones del corazón.

- Por la forma de la barriga (redondo niña y puntiagudo niño) (10)

- Según la luna se sabía el hijo siguiente: Creciente: diferente; en menguante: semejante al sexo del hijo anterior.

- Si se mueve mucho chico y si no, chica.

- Echar una cuchara (otros refieren una zapatilla o «espardeña») por detrás y si caía boca arriba era chico y boca abajo chica. bien).

- Según girara una llave (no aciertan a recordarlo

21.- ¿QUÉ ALIMENTACIÓN HAY QUE SEGUIR DURANTE EL EMBARAZO?

- Nada en especial, de lo que hubiera

en casa. $66,6 \%(20)$

- No comer picantes ni bebidas alcohólicas $6,6 \%$

- Dieta equilibrada, frutas, verduras y carne. $16,6 \%$

- No comer mucho pan para no engordar

- No comer grasas

- Chocolate para que diera fuerza en los días previos al parto

22.- ¿CREE QUE HAY QUE COMER PARA DOS? ¿POR QUÉ?

$-\mathrm{NO}$. $76,6 \%$

- SI.

$-\mathrm{NS} / \mathrm{NC}$ $16,6 \%$

Comentarios: $6,6 \%$

- No, los críos engordan encima del delantal, no debajo.

- No, porque ellos comen de la sangre de la madre.

- No, porque engorda la madre y el niño no nace mejor.

- No, pero se decía entonces que sí; con lo tuyo es suficiente. 
- No, aunque yo comía más porque tenía mucha hambre.

- Sí, porque me lo decía mi madre.

- Sí, porque el niño come de nosotras.

23.- ¿CREE QUE ES NECESARIO HACER REPOSO DURANTE EL EMBARAZO? ¿POR QUÉ?

- No, no es necesario. $80 \%$ (24)

- Sí, es conveniente $20 \%$

- Yo no lo he hecho nunca, seguía trabajando igual que antes

- No es necesario si no tienes problema

- Si trabajas estás más fuerte a la hora de empujar;

- No hace falta pero sería bueno.

- Sí, pero nunca lo hemos hecho

- Sí, porque estás más tranquila.

- Si no se mueven los niños nacen peor.

- Sí, si tienes peligro de abortar.

- Sí, por problemas en las piernas.

24.- ¿CONOCE ALGÚN MÉTODO PARA QUE NO SIGA ADELANTE UN ABORTO?

- No. NS/NC........................................ 66,6\% (20)

- Sí

- Reposo

25.- ¿CONOCE ALGÚN MÉTODO PARA PROVOCAR EL ABORTO?

- No, NS/NC.

- Sí:

- Comer albahaca.

- «Unas inyecciones», «unas pastillas»

- Saltar desde cierta altura, desde las escaleras

- «Fregarse las piernas y pies con mostaza y agua caliente»

- Meterse un tallo de perejil o algo por bajo, o hervir jabón e introducirlo con una pera en vagina, o aguja de punto.

- Comer perejil o tomarlo hervido, con azúcar por las mañanas.

- Tomar ciertas hierbas; hervir ciertas hierbas y tomar el caldio.

- Bañarse en agua fría, sobre todo los pies, o alternar agua fría y caliente.

- Lavarse con mucha frecuencia

- Pincharse dentro con una pluma de pavo

- Tomar una botella entera de «Agua Caravaña»

- Tirarse al agua fría desde una peña.

- Lavarse sus partes con vinagre hervido

- Hacer ejercicio fuerte.

26.- ¿CREE QUE SE PUEDEN MANTENER RELACIONES SEXUALES DURANTE EL EMBARAZO?

1.- Sí... $93,3 \%$

2.- No

3.- NS/NC

\section{¿POR QUE?}

$\mathrm{NO}$

- Se ríe y no contesta

- No, porque no conviene mover mucho la cosa

SI:

- Pero a mí no me apetecía.

- Sí, hasta el último día.

- Sí, a ellos les da lo mismo que la mujer esté embarazada

- Sí, mi marido no respetaba nada

- Sí, porque si no se van a buscar a otra.

- Sí, pero no tan a menudo y cuando estás avanzada

- Sí, porque hay que contentar al pariente

- Sí, estás más tranquila por no quedarte embazada

- Es normal, no pasa nada

- Sí, porque malo para el chiquillo no es.

- Sí, porque es bueno para nosotras

- Sí, pero a mí me daba miedo

- Sí, pero con conocimiento, y respetando que el parto estaba cerca.

- Sí, y bien a gusto.

27.- ¿CUÁL ES LA EDAD MÁS ADECUADA PARA EL EMBARAZO?

(Se ha agrupado por rangos de edades, para simplificar el análisis)

- De 15 a 19

$6,7 \%$

- De 20 a 25 . $66,7 \%(20)$

- Hasta los 35 años.

$16,6 \%$

- Da igual

$3,3 \%$

- Hasta que llega el retiro ..................... 3,3\%

- Cuanto más joven es mejor................ $13 \%$

28.- ¿HASTA QUÉ EDAD NO ES PELIGROSO QUE LA MUJER SE QUEDE EMBARAZADA?

- NS/NC. $13 \%$

- Hasta los 34-35 años.

- Hasta los 36-38 años.

$30 \%$

- Hasta los 40 años

$10 \%$

- Hasta los 45 $33,3 \%(10)$

- Hasta que se puede

$3,3 \%$

$6,6 \%$

- De 30 en adelante ya no nacen bien.

$3,3 \%$

29.- ¿CUÁNTO HAY QUE ESPERAR PARA TENER EL PRIMER HIJO?

- Según convenga a la pareja................ 13\%

- Yo los tuve en seguida ...................... 13\%

- Si lo desean, ¿para qué van a esperar? $3,3 \%$

- Disfrutar de la vida, sin pasarse ........

- 3 años o así

$16,6 \%$

- Según la edad a la que se casen.....

$6,6 \%$

- Cuando vienen vienen, cuando Dios quiera

- Ahora se puede programar y esperar, hay medios 


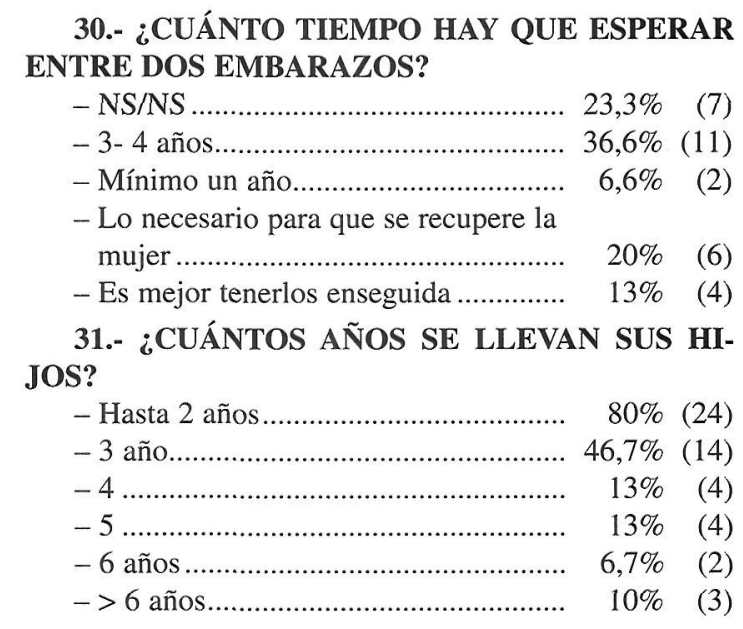

32.- ¿HA UTILIZADO ALGO PARA LAS MOLESTIAS DEL EMBARAZO?

- NO …………………………...... 83,3\% (25)

- SÍ................................................. 16,7\%

- «Comía garrofas mientras las recogía»

- «Leche condensada para los vómitos»

- No, porque yo es cuando mejor estaba

- Bicarbonato para el ardor de estómago

- Beber líquidos

III.C. Actitudes y creencias respecto al parto

33.- ¿CONOCE ALGÚN SANTO, VIRGEN O SANTUARIO A LOS QUE ACUDIR PARA TENER UN BUEN PARTO?

- No conocen a ninguno:

$46,7 \%$
$30 \%$
$3,3 \%$
$3,3 \%$
$3,3 \%$
$3,3 \%$
$3,3 \%$
$3,3 \%$
$3,3 \%$

- San Ramón Nonato:............................ 30 30\%

- Sagrado Corazón:................................. 3,3\%

- San Pascual ....................................... 3,3\%

- San Bartolomé ................................... 3,3\%

- Animas Benditas ................................. 3,3\%

- Virgen de la Asunción......................... 3,3\%

- Virgen de Lourdes............................... 3,3\%

- Cristo de Zalamea ............................. 3,3\%

En general las respuestas a esta pregunta han estado muy diversificadas, pese a que casi la mitad de las encuestadas afirman sin reservas «no conocer ninguno», repartiéndose el resto entre los reseñados.

\section{4.- ¿CONOCE ALGÚN MODO DE FACILITAR} EL PARTO?

- No conoce ningún modo $63,3 \%$

- Agachada y apoyada entre 2 sillas

- Colchón de pallorfas, en el suelo.

- Apretando la barriga

- Con ventosa

- Andando mucho y trabajando los días de antes

- Escoba (2 personas sujetaban el palo y la mujer hacía fuerza agachada, agarrada del mismo)
- Yendo al hospital

- Tomando cosas calientes.

- Encomendándose a Dios y a la Virgen

35.- ¿HA ESTADO HOSPITALIZADA PARA LOS PARTOS?

1.- Sí.......

$40 \%(12)$

2.- No .

3.- NS/NC

36.- SI HA ESTADO HOSPITALIZADA FUE EN:

1.- Hospital público $16,6 \%$

2.- Hospital privado

3.- Otros $16,6 \%$

4.- NS/NC $6,6 \%$

37.- ¿QUÉ PIENSA DE LOS PARTOS EN EL HOSPITAL?

¿QUÉ VENTAJAS Y DESVENTAJAS TIENEN?

-Mejor en el Hospital $80 \%$ (24)

Ventajas:

- Mejor atención

- Más medios y personal cualificado

- Mayor seguridad

- Más higiene

- Menos infecciones

- Rápida solución a las complicaciones

- Mejor en el domicilio

$20 \%$

- Tienes allí tus cosas

- No compartes habitación

- Está siempre la comadrona a tu lado

38.- ¿LE GUSTARIA MÁS EL PARTO DOMICILIARIO?

NO. $70 \%$

- Más riesgos

- Más complicaciones (hemorragias, desgarros, vueltas de cordón...)

- En casa se moría mucha gente.

SI. $30 \%$

- Miedo al Hospital

- Estar en tu medio habitual

- Más comodidades

- Allí no me siento como una enferma

- Mayor intimidad

39.- ¿CONOCE ALGÚN AMUleto O TALISMÁN PARA EL PARTO?

- No conoce

- Sí conoce. $73,3 \%$

- Estampa de San Ramón $26,6 \%$

- Estampa de San Pascual

- Estampa de San Francisco

- Medalla Virgen del Pilar.

- Medalla Virgen de la Asunción

- Cinta Rosa.

- Alguna estampita (no importa cuál). 
40.- ¿CONOCE SIGNOS DE GRACIA EN EL RECIÉN NACIDO?

- No conoce. $70 \%(21)$

- Conocen $30 \%$

- Oírlo llorar en el vientre, 5,

de las cuales:

- 3: no debe contarlo para que no se pierda

- 1: se debe contar para que se pierda la gracia $y$ en el futuro no sea curandero

- Nacer rodeado de membranas o dentro de la bolsa .

- Nacer en Viernes Santo.

- Si el niño tiene una «cruz» en el paladar

- Nacer de pie

41.- ¿CÓMO SE NOTA QUE VA A COMENZAR EL PARTO?

- Los dolores son más fuertes y seguidos cada vez $66,7 \% \quad 20)$

- Dolores y sangrado $10 \% \quad(3)$

- Dolores y rotura de bolsa $13 \% \quad(4)$

- Pesadez y pequeñas molestias $10 \% \quad(3)$

42.- ¿DE QUÉ FORMA SE EXPULSA LA PLACENTA Y EN QUÉ MOMENTO?

- Sale después de nacer el niño. El médico y/o la matrona aprietan la barriga hacia abajo para facilitar la expulsión

- Sale después del crío, pero yo no la he visto nunca $23,3 \%$

+ Otros:

- La comadrona o médico la observaban para ver si estaba bien

- ¡Yo de eso no me ocupaba!

- Estirando del cordón y apretando la barriga

- Soplando una botella

\section{3.- ¿QUÉ ES EL CORDON?}

- Lo que va enganchado al chiquillo y a la placenta (une madre e hijo $36,7 \%$ (11)

- Medio para alimentar al niño. $20 \% \quad(6)$

- Lo que cortan cuando nace el niño.... 16,6\%

- Una tripa.

$-\mathrm{NS} / \mathrm{NC}$

$13 \%$

\section{¿CUANDO HAY QUE CORTARLO?}

- Nada más nacer el niño. $93,3 \%(28)$

-Un rato después de nacer el niño.

$6,7 \%$
III.D.- Actitudes y creencias respecto al puerperio 44.- ¿CUANTOS DIAS DE REPOSO HAY QUE GUARDAR TRAS EL PARTO?

- 3-5 días en cama.......................... . . 23,3\% (7)

- Hasta que estabas bien (15-20 d) . . 16,6\% (5)

- En torno a la cuarentena ....... 16,6\% (5)

- 8 días en cama ............ 13,3\% (4)

- A los 2 días ya se levantaba...... 13,3\% (4)

- Ninguno ............... 10\% (3)

- Según sangrado.............

- Si estabas en el hospital, una semana

45.- ¿QUÉ CUIDADOS HAY QUE SEGUIR DURANTE LOS DÍAS SIGUIENTES AL PARTO?

- Chocolate, caldos de gallina y leche. $33,3 \%$ (10)

- Yo no tuve ninguno en especial ... . 33,3\% (10)

- Lavados (cuidar la herida de abajo y el cordón ............... 20\% (6)

- Ayuda de la familia en casa...... 10\% (3)

Otros:

- La comadrona te visitaba. ....... 2

- Tomar vino si se perdía mucha sangre 1

- No tocar a la de al lado, ni fregar, solo barrer

- No entrar al corral con olores .... . 1

- No lavarse mucho y reposar si se puede 1

- No hacer uso del matrimonio .... . 1

46.- ¿CREE QUE HAY QUE SEGUIR ALGÚN REGIMEN ESPECIAL EN LOS DÍAS SIGUIENTES AL PARTO?

- Caldos, para hacer leche y en general alimentarse bien y tomar más leche .......... 50\% (15)

- No comer ciertos alimentos (naranjas, tomates, olivas, helados, vino, cerdo, ni nada picante, ni salados). . . . . . . . . . . 16,6\%

- Estar encamada . . . . . . . . . . . . 2

- Lavado en cama, pero no el pelo... 2

- Cuidarse como de costumbre ..... o durante el emb............ 2

- Recibir visitas. . . . . . . . . . . . . 1

- Relaciones sexuales precoces, .... pero no en seguida $\ldots \ldots \ldots \ldots \ldots$

- Tomar vino para hacer buena leche .

47.- ¿CREE QUE LOS DÍAS POSTERIORES AL PARTO ES UN PERÍODO DIFÍCIL PARA LA MUJER? ¿POR QUÉ?

- No, para nada es un periodo difícil, porque..................

- No me dejaron sola nunca

- No es difícil, si llevas un poco de cuidado

- Depende de si tuviste algún problema en el parto

- Lo pasé mal, fue un periodo difícil por:

- Hay peligro de hemorragia, «El cuerpo está abierto» ............. 
- Falta de experiencia, necesitar ....

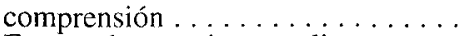

- Era una boca más para alimentar, . .

y más trabajo. . . . . . . . . . . .

- Miedo a quedar nuevamente embarazada 2

- Me quemaron la espalda con «espardeñas» calientes

- Depresión: estampé al niño porque lloraba.

- Te quedabas muy flojica porque estabas mal alimentada

- Se morían pocas mujeres, para los cuidados que tenían.

- «Una parturienta tiene los 40 días la tumba abierta $\gg$

\section{IV.- DISCUSIÓN DE RESULTADOS}

\section{Realización personal de la maternidad}

En nuestro estudio se detecta que mayoritariamente la respuesta fue afirmativa, en el sentido de que la maternidad realiza a la mujer como tal, ya que, incluso las mujeres que respondieron negativamente «de entrada», reconocían la conveniencia de ser madre para una mayor realización personal.

\section{Fecundidad y fertilidad (Preguntas 12 a 16 y 20)}

- En cuanto a quién es el responsable de no tener hijos en la pareja, en general manifiestan que «de los dos», es decir, que se acepta la implicación de ambos en este aspecto. Es importante matizar que cuando se afirma que «la culpa es de un miembro de la pareja» ésta siempre se le imputa a la mujer.

- Respecto a remedios para tener hijos cuando tardan en Ilegar, el $100 \%$ de las mujeres encuestadas admiten no conocer ninguno, aunque sugieren «paciencia», «tranquilidad». En solo un caso se nos ha comentado la existencia de una piedra «del olivino» en Lanzarote, que, engarzada como joya posee atributos en este sentido. Esta creencia se mantiene en la actualidad.

- En cuanto a la creencia respecto a santos o vírgenes a los que recurrir para la fecundidad, mayoritariamente (22/30) no conocen ninguno; en tres casos se nombró a San Ramón Nonato y se reparte el resto de respuestas en advocaciones particulares.

- La mayoría no cree en la influencia de los astros en la fecundidad; en algún caso se relaciona a la luna, pese a no poder determinar su papel en ello.

- El origen de los niños les es difícil de explicar a la mayoría; parece como si se le diera más importancia a la aportación masculina (papel activo) y la mujer como receptora. Ha llamado la atención el comentario relativo a que la formación del sexo masculino es más rápida que la del sexo femenino ( 40 días frente a 3 meses).
- 21 mujeres respondieron saber algún método para conocer el sexo del niño (aunque en general les daban poca credibilidad); por la riqueza de sus comentarios remitimos al lector al apartado «Resultados», pregunta 20.

\section{Antojos: (17-18-19)}

En general se acepta la existencia de los antojos, adquiriendo las formas tradicionales (flores, frutas, etc.) y sus repercusiones en el niño al nacer.

\section{Medidas higiénico-dietéticas durante el embarazo (alimentación, reposo, higiene, sexualidad, intervalo entre embarazos) (cuestiones 21-23, 26-32).}

Respecto a la alimentación se ha respondido que no precisaban cuidados especiales; además se ha desmitificado la idea de «comer para dos». El reposo se ha considerado como no necesario durante el embarazo (salvo situaciones especiales); a este fin cabe recordar que las mujeres de nuestro estudio pertenecen a una comarca en la cual la mujer ha compatibilizado sus embarazos con una vida profesional muy activa (ya fuera en el campo, taller o en la propia casa). Incluso ven como muy positivo realizar alguna actividad durante el embarazo. Para algunas mujeres las relaciones sexuales durante el embarazo se tenían «obligadas» para mantener satisfecho a su pareja, mientras que otras mujeres lo hablan como una experiencia más satisfactoria (para ambos) por poder disfrutar del acto sexual sin temor a un nuevo embarazo. Se acepta que los hijos deben tenerse a edades tempranas, pero no excesivamente (desde los 20 a los 30 años) situándose el límite peligroso por debajo de 15 y por encima de 35 , aproximadamente. El intervalo entre embarazos, más que determinarlo de manera numérica se fija en aspectos cualitativos como el estado de la madre, su recuperación, la independencia del niño, etc. Era infrecuente tener molestias durante el embarazo, por lo cual no tomaban nada y si aparecían lo vivían como algo natural.

\section{Aborto}

En nuestro estudio una gran parte de mujeres dicen no saber cómo evitar el aborto, aunque reconocen que el reposo es la única medida a adoptar. Respecto a cómo provocarlo las respuestas han sido muy diversas, aunque en general ellas mismas dudaban de la eficacia de los métodos y de las personas que los aplicaban. (Ver apartado de Resultados, preguntas 24 y 25 ).

\section{Actitudes y creencias respecto al puerperio}

Se repite el mismo patrón que en las actitudes y creencias respecto al embarazo: no se conoce ningún santo y cuando se refieren a alguno es mayoritariamente San Ramón Nonato o devociones particulares de la zona o ba- 
rrio. Para facilitar el parto se especifican técnicas concretas que utilizaron ellas mismas o sus madres (remitimos al lector al apartado Resultados, pregunta $n^{\circ} 34$ )

En cuanto a la hospitalización la mayoría (18/30) no lo estuvo, aunque en preguntas posteriores se refleja su predisposición a parir «si fuera hoy» en el medio hospitalario. No se aprecian diferencias significativas respecto a hospital público o privado, por disponer de mayores medidas de seguridad para madre y niño. Menos de una tercera parte dicen preferir el parto domiciliario.

- La presencia de talismán o amuleto en el momento del parto se admite en muy pocos casos, siendo además muy diversos (figuras sacras, cintas, piedras, etc.)

- La existencia de signos de gracia en el recién nacido se reconoce de manera escasa, de la que cabe destacar el «oír llorar al feto en el vientre materno».

- El inicio del parto se relaciona mayoritariamente con la presencia del DOLOR. La expulsión de la placenta era posterior y si se retrasaba citaban la «expresión del útero» por la matrona. Una afirmación curiosa es la de «soplar una botella» para facilitar la expulsión. En la mayoría de los casos las mujeres no mostraron interés en verla. Conocen la existencia del cordón y el momento de cortarlo, pero no saben explicar muy bien su función.

\section{Actitudes y creencias respecto al puerperio}

Sobre el periodo de reposo post-parto no existe unanimidad; se aprecia la misma tónica que en los cuidados durante el embarazo («sólo si surgen complicaciones, sangrado, etc». Cabe destacar la importancia del caldo de gallina y productos energéticos (chocolate, leche ) para la recuperación de las fuerzas. Llama la atención los comentarios respecto a la prevención de infecciones (evitar ciertas tareas domésticas y agrícolas), aunque un tercio admite no haber tenido cuidados especiales. Lo anterior refuerza la opinión de que el puerperio no era un periodo especialmente difícil para la mujer.

\section{V.- CONCLUSIONES}

Las mujeres de nuestro estudio tienen en común:

- Gran número de embarazos

- Otorgan gran importancia al tema de la maternidad

- Dedicación mayoritaria a «sus labores» o ayudando en ciertas tareas al esposo.

- La mayoría han tenido partos domiciliarios.

- En la actualidad les inspira más confianza el parto hospitalario.

- Son partidarias de que exista un distanciamiento entre los embarazos pese a que ellas no los han utilizado.

- No han recibido controles sanitarios durante su embarazo.
- Hacen referencia a la matrona como la profesional de la asistencia al parto y al puerperio, y de la atención al niño en este periodo.

- No creen en la influencia de los astros en este proceso vital ni manifiestan creencia en símbolos religiosos que favorezcan el mismo.

- El nivel educativo es bajo o muy bajo.

- Sobre los métodos abortivos se refieren a ellos de forma anecdótica, dudando de la eficacia de los mismos.

De todos modos, creemos que la mayor riqueza del estudio estriba, precisamente, en haber escuchado sus palabras, frases que han sido transcritas literalmente para garantizar la riqueza de sus comentarios.

\section{VI.- AGRADECIMIENTOS}

Nuestro más sincero agradecimiento a las treinta mujeres que atendieron nuestra petición y accedieron a compartir sus experiencias con nosotras (para muchas de ellas bastante lejanas en el tiempo), esforzándose en recordar el mayor número posible de detalles para enriquecer nuestro trabajo.

A nuestros familiares que pacientemente han soportado nuestras ausencias en la realización de este trabajo.

A nuestra profesora Rosa Ballester que nos ha iniciado en esta apasionante disciplina, aunque hayamos tenido que dedicar gran parte de nuestro tiempo.

\section{BIBLIOGRAFÍA}

Balaguer et al (1991) Fenómenos Biológicos y Fenómenos Culturales: La interpretación popular del ciclo vital en dos Comunidades Alicantinas. Monografías Sanitarias. Serie D., 10. Generalitat Valenciana. Consellería de Sanitat y Consum. IVESP, Valencia.

Consellería de Sanitat y Consum (1991) Libro Blanco de la Salud en la Comunidad Valenciana. Generalitat Valenciana, Valencia.

Fernández del Castillo, I. (1994) La revolución del nacimiento. Edaf, Madrid

Foster, GM (1980) Folklore y costumbres del embarazo, nacimiento e infancia. En La antropología médica en España (Kenny, M. y De Miguel J.M: eds), Anagrama, Madrid pp 249-265

Gómez Estebán, R.; Amilibia, S. (1985) Mujer, deseo y embarazo. Fundamentos, Madrid.

Limón, A. ; Castellote, E. (1980) La medicina popular en torno al embarazo y al parto al principio de siglo. En Antropología médica en España (Kenny, M. y De Miguel, J.M. eds). Anagrama, Madrid pp 227-248. 